\title{
Analysis of Speed Control of Separately Excited DC Motor Using FOPID with LQR
}

\author{
Anand Mickky ${ }^{1}$, Pratibha Tiwari ${ }^{2}$ \\ M.Tech (CI), EE, SHIATS, Allahabad, India ${ }^{1}$ \\ Assistant Professor, EE, SHIATS, Allahabad, India ${ }^{2}$
}

\begin{abstract}
This paper presents a simulation and performance analysis of speed control of brushless DC motor using FOPID with LQR. DC motor is widely used in industries even if its maintenance cost is higher than the induction motor. Speed control of DC motor is attracted considerable research and several methods are evolved. The LQR controller is the very commonly used compensating controller. This paper presents a comparison of time response specification between conventional Fractional order Proportional- Integral-Derivatives (FOPID) controller and Linear Quadratic Regulator (LQR) for a speed control of a separately excited DC motor. A class of fractional order systems having single non-integer order element which show highly sluggish and oscillatory open loop responses have been tuned with an LQR based FOPID controller. The goal is to determine which control strategy delivers better performance with respect to DC motor's speed. Performance of these controllers has been verified through simulation using MATLAB/SIMULINK software package. According to the simulation results, liner quadratic regulator method gives the better performance, such as settling time, steady state error and overshoot compared to FOPID controller. This shows the superiority of liner quadratic regulator method over FOPID controller.
\end{abstract}

Keywords: Linear Quadratic Regulator (LQR) Proportional-Integral Derivative Controller, FOPID controller, DC motor speed control.

\section{INTRODUCTION}

DC motor is a power actuator which converts electrical order proportional-integral derivative (FOPID) controller, energy into mechanical energy. DC motor is used in I and D operations are usually of fractional order; Application where wide speed ranges are required. The therefore, besides setting the proportional, derivative and greatest advantage of dc motors may be speed control. The integral constants KP, KI, KD, we have two more term speed control stand for intentional speed variation parameters; the order of fractional integration $\lambda$ and that of carried out manually or automatically. DC motors are most fractional derivative $\boldsymbol{\mu}$. Determining an optimal set of suitable for wide range speed control and are therefore value for a given process plant. This has been solved by used in many adjustable speed drives Because of their high using N-integer tool box. The values of KP, KI, KD, $\lambda$ and reliabilities, flexibilities and low costs, DC motors are $\boldsymbol{\mu}$ are selected by hit and trial method.

widely used in industrial applications, robot manipulators and home appliances where speed Control of motor is required. Since speed is directly proportional to armature voltage and inversely proportional to magnetic flux produced by the poles, adjusting the armature voltage and/or the field current will change the rotor speed. DC motors have been widely used in many industrial applications such as electric vehicles, steel rolling mills, electric cranes, and robotic manipulators due to precise, wide, simple, and continuous control characteristics. Proportional-Integral-Derivative controller has been used for several decades in industries for process control applications. At the same time FOPID controller has some disadvantages namely; the undesirable speed overshoot, the sluggish response due to sudden change in load torque and the sensitivity to controller gains KI and KP. The performance of this controller depends on the accuracy of system models and parameters. Therefore there is need of a controller which can overcome disadvantages of PID controller. DC motor can also be controlled by a nonconventional control technique known as FOPID control, a generalized version of integer order control. Dynamic systems based on fractional order calculus have been a subject of extensive research in recent years. In fractional

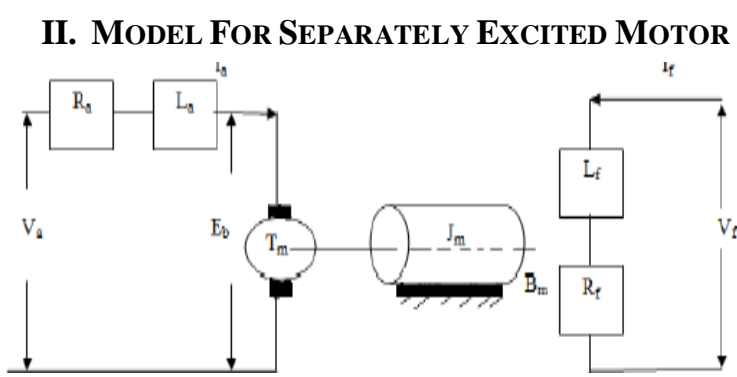

Fig-1 Separately excited DC motor model

Assuming constant field excitation the armature circuit electrical equation is written as:

$$
\begin{aligned}
& \mathrm{Va}=\mathrm{Eb}+\mathrm{IaRa}+\mathrm{La}(\mathrm{dIa} / \mathrm{dt}) \ldots \\
& \mathrm{Tm}=\mathrm{Jmd} \omega \mathrm{dt}+\mathrm{Bm} \omega+\mathrm{TL}
\end{aligned}
$$

Where

* Va is the armature voltage (in volt)

$\mathrm{Eb}$ is back emf the motor (in volt)

Ia is the armature current (in ampere)

$\mathrm{Ra}$ is the armature resistance (in ohm)

$\mathrm{La}$ is the armature inductance (in Henry)

$\mathrm{Tm}$ is the mechanical torque developed (in $\mathrm{Nm}$ ) 
* Jm is moment of inertia (in $\mathrm{kg} / \mathrm{m}^{2}$ )

* Bm is friction coefficient of the motor (in $\mathrm{Nm} /$ $(\mathrm{rad} / \mathrm{sec}))$

* $\omega$ is angular velocity (in $\mathrm{rad} / \mathrm{sec}$ )

* Ta $=$ Armature Time Constant, $\mathrm{Ta}=\mathrm{La} / \mathrm{Ra}$

After simplifying the above equations, the overall transfer function is obtained as shown in Fig. 2.

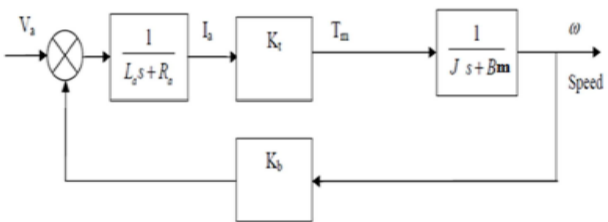

Fig-2 Block diagram of separately excited DC motor model

$$
G(s)=\frac{\omega(s)}{V_{a}(S)}=\frac{K_{T}}{\left(L_{a} * j\right) S^{2}+S\left(R_{a} * j+L_{a} * B\right)+\left(R_{a} * B+K_{b} * K_{T}\right)}
$$

Parameters of separately excited DC motor model:

\begin{tabular}{cl}
\hline Symbol & Magnitude \\
\hline Ra & $0.5 \Omega$ \\
La & $0.02 \mathrm{H}$ \\
Va & $200 \mathrm{~V}$ \\
Jm & $0.1 \mathrm{Kg} \cdot \mathrm{m} 2$ \\
$\mathbf{B m}$ & $0.008 \mathrm{~N} \cdot \mathrm{m} / \mathrm{rad} / \mathrm{sec}$ \\
\hline $\mathbf{k}$ & $1.25 \mathrm{~V} / \mathrm{rad} / \mathrm{sec}$ \\
\hline
\end{tabular}

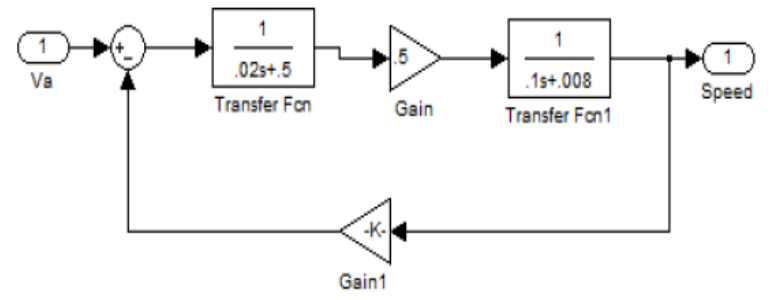

Fig. 3 Block Diagram of SEDC Motor model

\section{iII.Fractional Order PID Controller}

To study under the fractional order controllers, the starting point is of course the fractional order differential equations using fractional calculus. Fractional calculus extends the classic concepts of differential and integral calculus to an arbitrary order.

The classical PID controller can be generalized into a fractional order PID controller, the so called PI $\lambda \mathrm{D} \mu$, whose integro-differential equation can be expressed as:All paragraphs must be justified, i.e. both left-justified and right-justified.

$\mathrm{ut}=\mathrm{Kpet}+1 / \mathrm{TlD}-\lambda$ et $-\lambda$ et $+\mathrm{TdD} \mu \mathrm{e}(\mathrm{t})$

Where KP is the proportional gain, TI is the integral time constant, TD is the derivative time constant, $\boldsymbol{\lambda}$ is the (noninteger) order of the integrator and $\mu$ is the (non-integer) order of the derivative action. The corresponding transfer function is expressed as

$$
\mathrm{CS}=\mathrm{U}(\mathrm{S}) / \mathrm{E}(\mathrm{S})=\mathrm{KP}(1+1 \mathrm{TIS} \lambda+\mathrm{TAS} \lambda)
$$

$\mathrm{KI}=\mathrm{KP} / \mathrm{TI}$ and $\mathrm{KD}=\mathrm{KP} \cdot \mathrm{TD}$,

we obtain $\mathrm{Cs}=\mathrm{KP}+\mathrm{KI} / \mathrm{S} \lambda+\mathrm{Kds} \lambda$

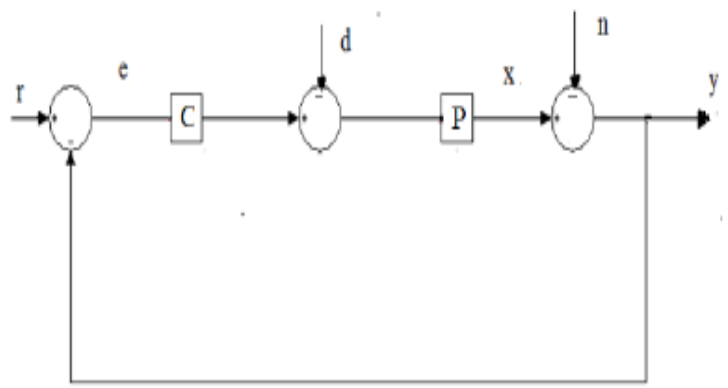

Fig.4 control scheme of FOPID

For the purpose of the description made in the following sections, denote as $\mathrm{S}$ the sensitivity function $\mathrm{S}(\mathrm{s})=1 / 1+\mathrm{C}$ $\mathrm{S} P(\mathrm{~S}) \ldots \ldots \ldots$ (6) As $\mathrm{T}$ the complementary sensitivity function $\mathrm{T} \mathrm{s}=\mathrm{C}(\mathrm{s}) \mathrm{P}(\mathrm{s}) / 1+\mathrm{C}(\mathrm{s}) \mathrm{P}(\mathrm{s}) \ldots \ldots$.... (7) And as $\mathrm{L}$ the open-loop transfer function $\mathrm{L}(\mathrm{s})=\mathrm{C}(\mathrm{s}) \mathrm{P}(\mathrm{s})$.

\section{IV.LINEAR QUADRATIC REGULATOR}

Linear quadratic regulator design technique is well known in modern optimal control theory and has been widely used in many applications. LQR is a method in modern control theory that used state-space approach to analyse such a system. Using state space methods it is relatively simple to work with Multi- Input Multi-Output (MIMO) system. Using state space methods it is relatively simple to work with Multi- Input Multi-Output system. LinearQuadratic Regulator (LQR) optimal control problems have been widely investigated in the literature. The performance measure is a quadratic function composed of state vector and control input. If the linear time-invariant system is controllable, the optimal control law will be obtained via solving the algebraic Ricci equation optimal control. The function of Linear Quadratic Regulator (LQR) is to minimize the deviation of the speed of the motor. The speed of the motor is specifying that will be the input voltage of the motor and the output will be compare with the input. In general, the system model can be written in state space equation as follows:

$$
\mathrm{X}=\mathrm{Ax}+\mathrm{Bu}
$$

$A$ is the state matrix of order $n \times n ; B$ is the control matrix of order $\mathrm{n} \times \mathrm{m}$. Also, the pair $(\mathrm{A}, \mathrm{B})$ is assumed to be such that the system is controllable. The linear quadratic regulator controller design is a method of reducing the performance index to a minimize value. The minimization of it is just the means to the end of achieving acceptable performance of the system. For the design of a linear quadratic regulator controller, the performance index $(J)$ is given by:

$$
J=\int_{0}^{\infty}\left(x^{T} Q_{x}+u^{T} R_{u}\right) d t
$$


Where $Q$ is symmetric positive semi-definite state weighting matrix of order $\mathrm{n} \times \mathrm{m}$ and $\mathrm{R}$ is symmetric positive definite control weighting matrix of order $\mathrm{n} \times \mathrm{m}$ .The choice of the element $\mathrm{Q}$ and $\mathrm{R}$ allows the relative weighting of individual state variables and individual control inputs as well as relative weighting state vector and control vector against each other.

\section{Simulation \& Result}

In order to verify the validity of the linear quadratic regulator controller, several simulation tests are carried out using MATLAB/SIMULINK software package. The performance of linear quadratic regulator controller has been investigated and compared with the FOPID controller.

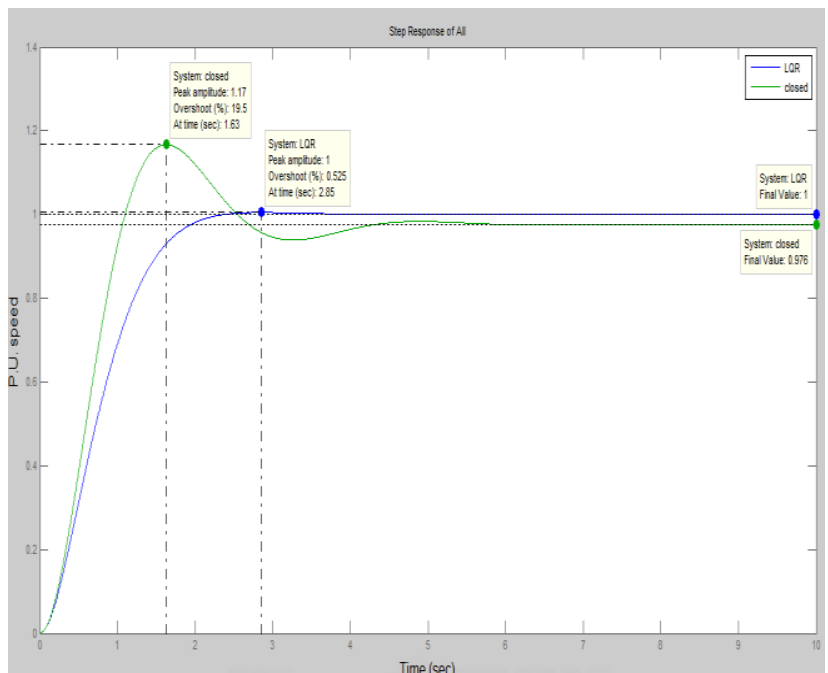

Fig-5 Graph between P.U speed Vs time response among LQR system

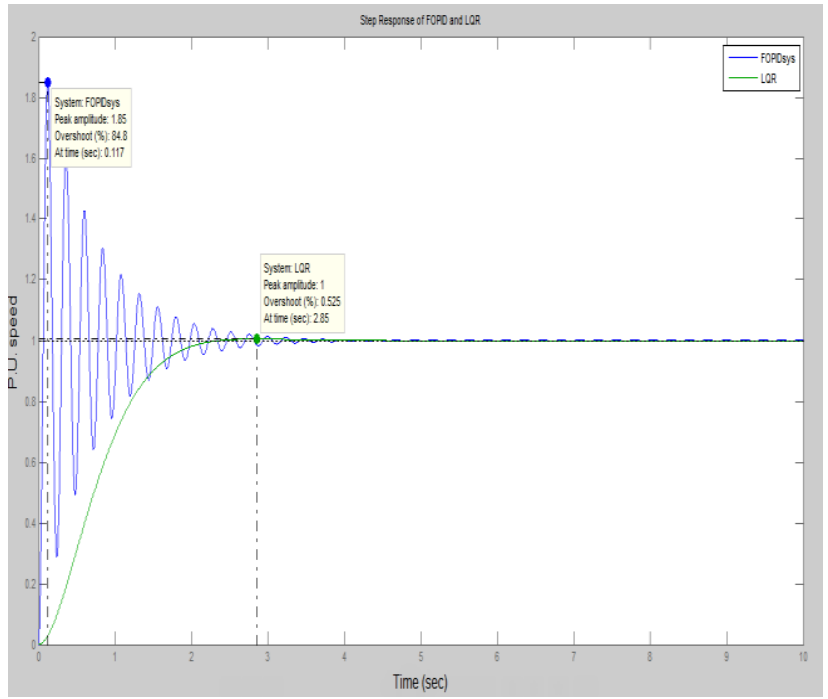

Fig-6 Graph between P.U speed Vs time response of FOPID with LQR system

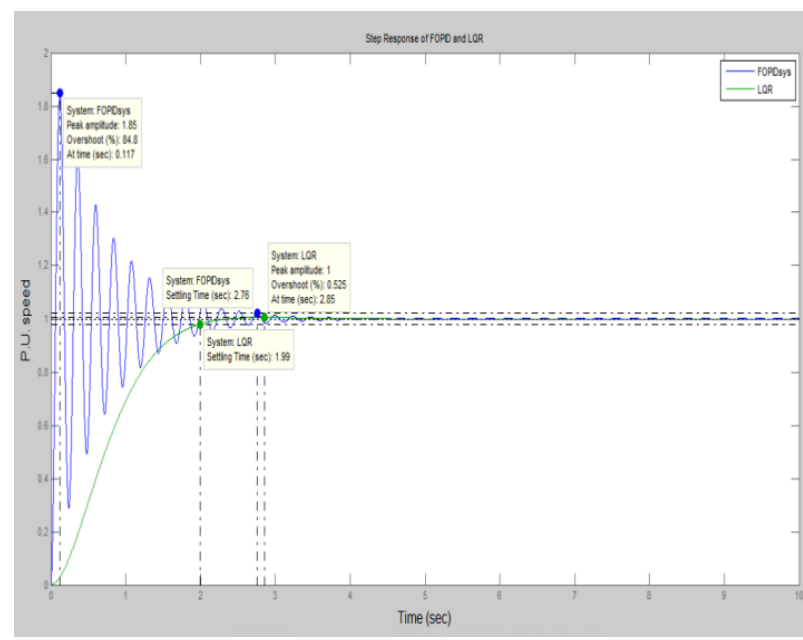

Fig-7 Graph between P.U speed Vs time response of FOPID with LQR system

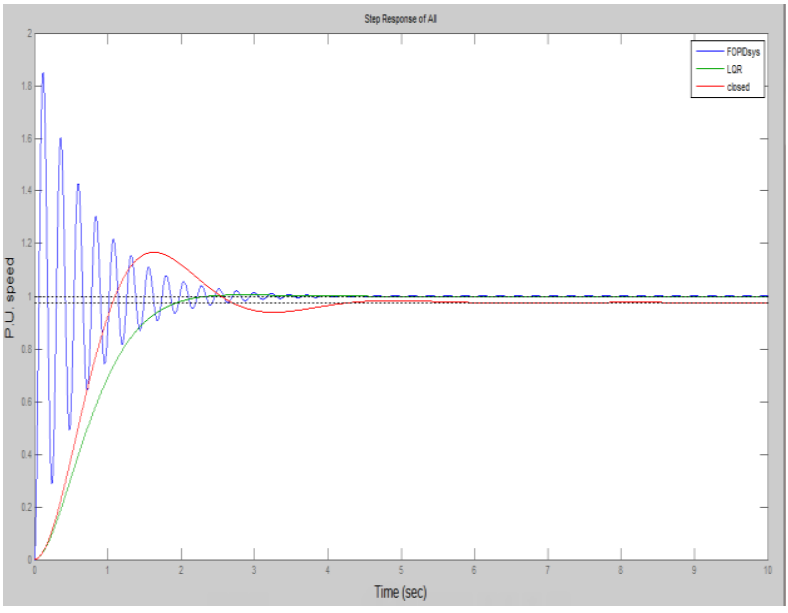

Fig-8Graph between P.U speeds Vs time responses using FOPID with LQR system

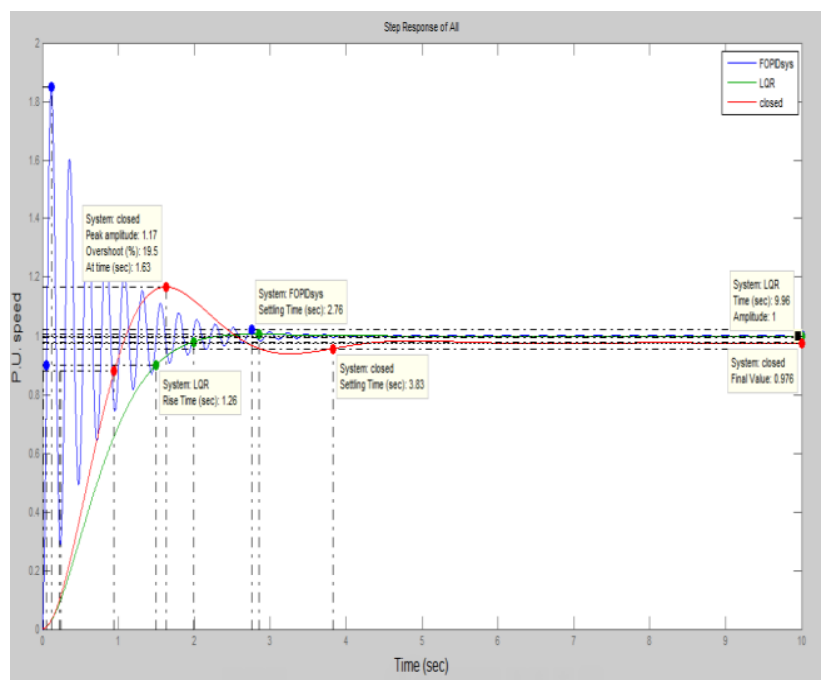

Fig-9 Graph between P.U speeds vs time responses of speed control using FOPID with LQR system 


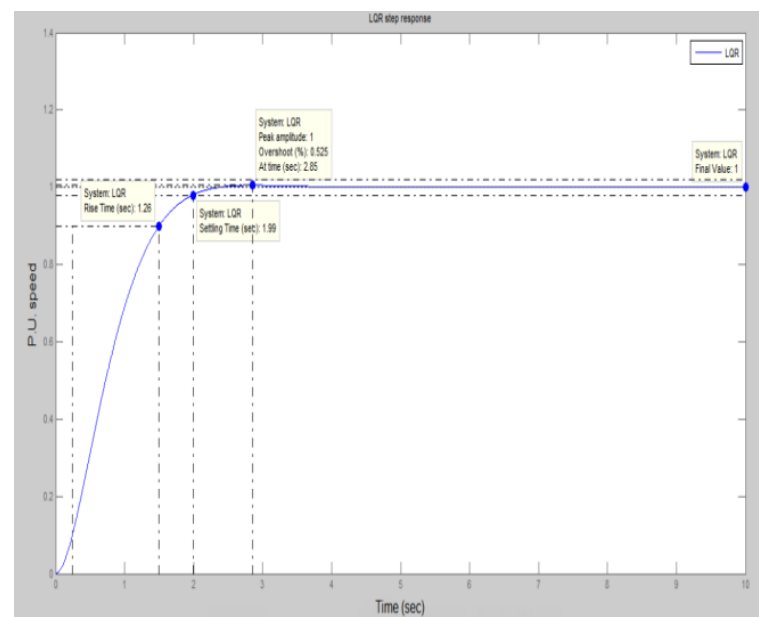

Fig-10 Graph between P.U speeds vs time responses with LQR system

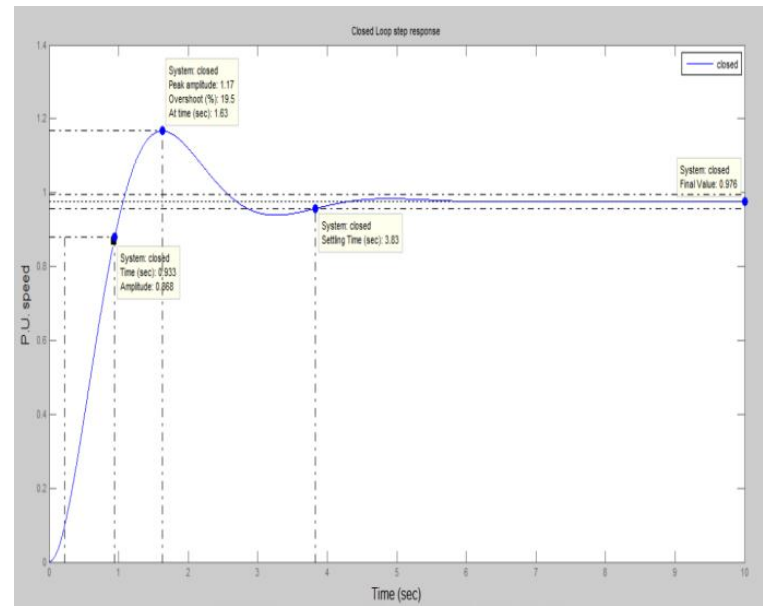

Fig-11 System Response with LQR controller

\section{CONCLUSION}

In this paper the speed of a DC motor is controlled using FOPID via Linear-Quadratic Regulator (LQR). The simulation results are obtained using MATLAB/SIMULINK. Optimal LQR strategy and FOPID controller have been considered in this paper for controlling the speed of a separately excited DC motor. The performance of the two controllers is validated through simulations. A number of simulation results are presented for comparison. Based on the comparative simulation results, one can conclude that the linear quadratic regulator controller realizes a good dynamic behavior of the separately excited DC motor with a rapid settling time, no overshoot, and zero steady state error compared to FOPID controller under nominal condition. The results show that the overshoot, settling time, peak time and control performance has been improved greatly by using Linear-Quadratic Regulator (LQR) controller. The LQR controller has more advantages, such as higher flexibility, control, better performance compared with FOPID. Hence, the comparison results showed that the Linear-Quadratic Regulator (LQR) controller was the best controller. 